\title{
Comparative analyses of postoperative complications and prognosis of different surgical procedures in stage II endometrial carcinoma treatment
}

\author{
This article was published in the following Dove Press journal: \\ OncoTargets and Therapy \\ 16 February 2016 \\ Number of times this article has been viewed
}

\author{
Hongmei Yin' \\ Ting Gui ${ }^{2}$ \\ 'Department of Obstetrics and \\ Gynecology, Binzhou Medical \\ University Hospital, Binzhou Medical \\ University, Binzhou, Shandong, \\ ${ }^{2}$ Department of Obstetrics and \\ Gynecology, Peking Union Medical \\ College Hospital, Peking Union \\ Medical College, Chinese Academy \\ of Medical Sciences, Beijing, \\ People's Republic of China
}

\begin{abstract}
Objective: To investigate the impact of surgical resection extent on the postoperative complications and the prognosis in patients with stage II endometrial cancer.

Methods: A total of 54 patients were retrospectively reviewed, 35 patients underwent subradical hysterectomy and 19 patients received radical hysterectomy, both with simultaneous bilateral salpingo-oophorectomy and pelvic and paraaortic lymphadenectomy.

Results: Comparing the surgical outcomes in subradical hysterectomy group vs radical hysterectomy group, there were no significant differences in operative time, estimated blood loss, and hospital stay. After surgery, $37.1 \%$ vs $36.8 \%$ patients received postoperative radiotherapy in the subradical hysterectomy group vs radical hysterectomy group, without statistically significant difference. As for postoperative complications, the early postoperative complication rate in patients who underwent subradical hysterectomy was $14.3 \%$, significantly lower than that in patients submitted to radical hysterectomy ( $14.3 \%$ vs $42.1 \%$ ), with $P=0.043$. However, there was no significant difference in late postoperative complication rate between the two surgical procedures. Regarding the clinical prognosis, patients receiving the subradical hysterectomy showed similar survival to their counterparts undergoing the radical procedures. The relapse rate was $5.71 \%$ vs $5.26 \%$, respectively, without significant difference. There were no deaths in both surgical groups.

Conclusion: For stage II endometrial carcinoma, subradical hysterectomy presented with less early postoperative complications and similar survival duration and recurrence compared with radical hysterectomy and should be advocated in clinical treatment.
\end{abstract}

Keywords: endometrial carcinoma, stage II, postoperative complication, prognosis, surgical extent

\section{Introduction}

Endometrial carcinoma is one of the three most common gynecologic malignancies. ${ }^{1}$ While treatment is often multimodality, the primary strategy is surgery. ${ }^{2}$ Traditionally, surgery has been performed using an abdominal approach (laparotomy); however, with the advent of laparoscopy, in the past decade, it became feasible to carry out the procedure using a minimally invasive technique. ${ }^{3,4}$ Several studies have demonstrated that laparoscopy could result in fewer postoperative adverse events and shorter hospitalization than laparotomy, with no significant difference in the overall survival. ${ }^{5,6}$

According to the update of International Federation of Gynecology and Obstetrics (FIGO) staging system in 2009, endometrial carcinoma at stage II refers to tumor invasion of cervical stroma. Surgical procedures mainly include laparoscopic hysterectomy, 
adnexectomy, and retroperitoneal lymphadenectomy. ${ }^{7}$ However, the surgical dissection extent of hysterectomy is still controversial. Both subradical hysterectomy and radical hysterectomy have been widely used in clinic. Does radical hysterectomy have better therapeutic effects than subradical hysterectomy? Are there any significant differences in postoperative complications, such as urinary retention, urinary tract infection, pelvic lymphocyst, deep venous thrombosis, neuropathy, and subcutaneous hematoma, between the two surgical procedures? Which procedure should we prefer in clinic?

Accordingly, by retrospectively reviewing patients with stage II endometrial carcinoma who received either subradical or radical hysterectomy, we sought to make comparative analyses of the prognosis and the related postoperative complications in patients to explore the optimal surgical procedure, in the hope of providing some evidence in avoiding excessive surgery in endometrial carcinoma treatment.

\section{Methods}

Our research was approved by the Ethics Committee of Binzhou Medical University Hospital, and written informed consent from each patient was obtained before treatment.

\section{Patient information}

Between July 2009 and December 2014, 54 patients with stage II endometrial carcinoma received surgical treatment in the Department of Obstetrics and Gynecology at Binzhou Medical University Hospital. Data pertaining to patient demographics, diagnosis, operative outcomes, postoperative complications, clinical prognosis, and follow-up were collected from the medical records and the clinical database for retrospective review.

\section{Inclusion criteria}

The inclusion criteria were as follows:

1. Patients who were diagnosed with endometrial carcinoma by pathological examination after fractional diagnosis and curettage.

2. Patients who did not receive radiotherapy, chemotherapy, or hormone therapy before the surgery.

3. Patients whose follow-up was reliable.

\section{Surgery and adjuvant therapy}

All the patients were diagnosed by pathological review after fractional diagnosis and curettage. Comprehensively considering patient age, medical complications, and magnetic resonance imaging (MRI) examination, we preliminarily determined the tumor invasion extent. For tumors suspicious for invasion of cervical stroma after preoperative assessment, we classified them as stage II endometrial carcinomas. In clinic, two surgical procedures existed, subradical hysterectomy or radical hysterectomy, with simultaneous bilateral adnexectomy and pelvic and paraaortic lymph nodes dissection. All surgeries were performed by surgeons who were experienced and proficient in advanced laparoscopic gynecologic procedures. The total operative time was recorded as the time from the first skin incision to the last port site skin closure. The estimated blood loss and postoperative hospital stay were also recorded. Postoperative complications were divided into early (less than postoperative 8 weeks) and late (beyond postoperative 8 weeks) events. ${ }^{8}$ Adjuvant radiotherapy was given in the presence of high-risk factor for recurrence, such as invasion of deep muscularis, poor differentiation, and lymph vascular space involvement.

\section{Follow-up}

All the patients had regular follow-up, and the period was considered from the day of surgery to July 31, 2015. A routine medical check-up, including symptoms inquiry (vaginal bleeding, pelvic or lumbar ache, cough, shortness of breath, ascites, edema of lower extremities, etc), an abdominal and gynecological examination, tumor markers, and ultrasound of the pelvis and abdomen, was performed at each follow-up visit. The follow-up plan in our hospital was as follows: every 3 months for the first 2 years, every 6 months for the third to fifth years, and once per year thereafter.

\section{Statistical analysis}

Statistical analysis was performed by SPSS software. Fisher's exact test was used to analyze the categorical data. Kaplan-Meier analysis was used to compare the overall survival. A $P$-value $<0.05$ was considered to be statistically significant.

\section{Results \\ Clinicopathological characteristics of patients}

During the 6 years of our study, 216 patients were suspicious of cervical stroma invasion by MRI examination and 54 patients were confirmed as stage II endometrial carcinoma after surgical-pathological staging. There were 35 and 19 patients who underwent subradical hysterectomy and radical hysterectomy, respectively, with a median age of 55 years (range 37-72 years) and 53 years (range 40-66 years). The ratio of endometrioid carcinoma 
Table I Clinicopathological characteristics of patients

\begin{tabular}{lll}
\hline Clinicopathological features & Subradical hysterectomy & Radical hysterectomy \\
\hline Number & 35 & 19 \\
Age, years: median (range) & $55(37-72)$ & $53(40-66)$ \\
Endometrioid carcinoma & $82.8 \%(29 / 35)$ & $84.2 \%(16 / 19)$ \\
Surgical-pathological stage & Stage II (I00\%) & Stage II (I00\%) \\
Tumor grade & $18(51.4 \%)$ & $10(52.6 \%)$ \\
$\quad$ Well differentiated & $13(37.1 \%)$ & $6(31.5 \%)$ \\
$\quad$ Moderately differentiated & $4(11.4 \%)$ & $3(15.7 \%)$ \\
$\quad$ Poorly differentiated & & $15(78.9 \%)$ \\
Muscularis invasion & $27(77.1 \%)$ & $4(21.1 \%)$ \\
$\quad$ Superficial muscularis & $8(22.9 \%)$ & $2(10.5 \%)$ \\
$\quad$ Deep muscularis & $3(6.5 \%)$ & $17(89.5 \%)$ \\
$\quad$ Intravascular tumor emboli & $32(91.4 \%)$ & \\
$\quad$ Positive & & \\
\hline
\end{tabular}

Note: Data presented as number of patients (\%) unless stated otherwise.

was $82.8 \%$ (29/35) vs $84.2 \%$ (16/19), respectively. Regarding postoperative pathological examination, $11.4 \%$ vs $15.7 \%$ patients were poorly differentiated, $22.9 \%$ vs $21.1 \%$ patients had deep muscularis invasion, and $91.4 \%$ vs $89.5 \%$ patients were found to have intravascular tumor emboli in the subradical hysterectomy group vs radical hysterectomy group, respectively. No significant differences were observed in age, histological type, and high-risk factors (tumor grade, muscularis invasion, and intravascular tumor emboli) for recurrence after statistical analyses (Table 1).

\section{Surgical outcomes and postoperative radiotherapy}

No significant differences were observed in operative time (174 minutes vs 185 minutes), estimated blood loss (625 mL vs $660 \mathrm{~mL}$ ), and hospital stay (12 days vs 13 days) in the subradical hysterectomy group vs radical hysterectomy group. Postoperative radiotherapy was given in the presence of high-risk factors for recurrence, such as poor differentiation, invasion of deep muscularis, and positive intravascular tumor emboli. Our data revealed that $37.1 \%$ vs $36.8 \%$ patients received postoperative radiotherapy in the subradical hysterectomy group vs radical hysterectomy group, respectively, without statistically significant difference (Table 2).

Table 2 Surgical outcomes according to the procedures

\begin{tabular}{lll}
\hline Variables & $\begin{array}{l}\text { Subradical surgery } \\
(\mathbf{n}=\mathbf{3 5})\end{array}$ & $\begin{array}{l}\text { Radical surgery } \\
(\mathbf{n}=\mathbf{1 9 )}\end{array}$ \\
\hline Operative time (minutes) & 174 & 185 \\
Estimated blood loss $(\mathrm{mL})$ & 625 & 660 \\
Hospital stay (days) & 12 & 13 \\
Postoperative radiotherapy* & 13 & 7 \\
\hline
\end{tabular}

Note: *Data presented as number of patients.

\section{Postoperative complications}

The early complications occurring before postoperative 8 weeks included urinary retention $(n=3)$, urinary tract infection $(n=2)$, pelvic lymphocyst $(n=3)$, deep venous thrombosis $(n=1)$, subcutaneous hematoma $(n=1)$, hemovac site dehiscence $(n=1)$, and neuropathy $(n=2)$. The frequencies of late postoperative events were similar to those in the early postoperative period, including pelvic lymphocyst $(n=9)$, leg weakness $(n=2)$, and vaginal dryness $(n=2)$ (Table 3$)$.

The overall early complication rate was $24.1 \%$, with $14.3 \%$ vs $42.1 \%$ for the subradical procedures vs radical procedures, respectively, showing significant difference between the two groups with $P=0.043$. The overall late complication rate was $24.1 \%$, with $20.0 \%$ vs $31.5 \%$ for the subradical procedures vs radical procedures, respectively. However, there was no statistically significant difference between the two surgical procedures.

\section{Clinical outcomes}

Comparing the survival duration, the patients receiving the radical hysterectomy showed similar survival to their counterparts who received the subradical procedures, without significant difference (Figure 1 and Table 4).

Among the 54 patients, three patients relapsed, two patients in the subradical procedure group and one patient in the radical procedure group. The relapse sites in the subradical procedure group included pelvic cavity, inguinal lymph node, and liver. The relapse sites in the radical procedure group were pelvic cavity, omentum majus, and lung. The relapse rate was $5.71 \%$ vs $5.26 \%$ for the subradical procedure vs the radical procedure, respectively. Statistical analysis showed no significant difference between the two groups. There were no deaths in both surgical groups. 
Table 3 Early and late postoperative complications

\begin{tabular}{|c|c|c|c|c|}
\hline Variables & Total number & $\begin{array}{l}\text { Subradical } \\
\text { hysterectomy }(n=35)\end{array}$ & $\begin{array}{l}\text { Radical } \\
\text { hysterectomy }(n=19)\end{array}$ & $P$-value \\
\hline Early postoperative complications & $13(24.1 \%)$ & $5(14.3 \%)$ & $8(42.1 \%)$ & 0.043 \\
\hline Urinary retention & 3 & I & 2 & \\
\hline Urinary tract infection & 2 & I & 1 & \\
\hline Pelvic lymphocyst & 3 & I & 2 & \\
\hline Deep venous thrombosis & 1 & 0 & I & \\
\hline Subcutaneous hematoma & 1 & 0 & I & \\
\hline Hemovac site dehiscence & 1 & I & 0 & \\
\hline Neuropathy & 2 & I & 1 & \\
\hline Late postoperative complications & $13(24.1 \%)$ & $7(20.0 \%)$ & $6(31.5 \%)$ & 0.506 \\
\hline Pelvic lymphocyst & 9 & 5 & 4 & \\
\hline Leg weakness & 2 & I & I & \\
\hline Vaginal dryness & 2 & I & 1 & \\
\hline
\end{tabular}

\section{Discussion}

Surgery is the preferred treatment modality for endometrial carcinoma. As the prevalence of overweight and obesity continues to rise, an increased incidence of endometrial cancer is expected to be seen. In addition, overweight and obese women are also at risk for increased medical comorbidities, including diabetes and cardiovascular diseases. ${ }^{9}$ Therefore, there has been more and more interest in identifying surgical techniques that may allow for adequate surgical treatment for patients while minimizing surgical morbidity and mortality.

In the past decade, increasingly, gynecologic oncologists were offering laparoscopy in treating patients with endometrial cancers. Choosing surgical procedure was dependent

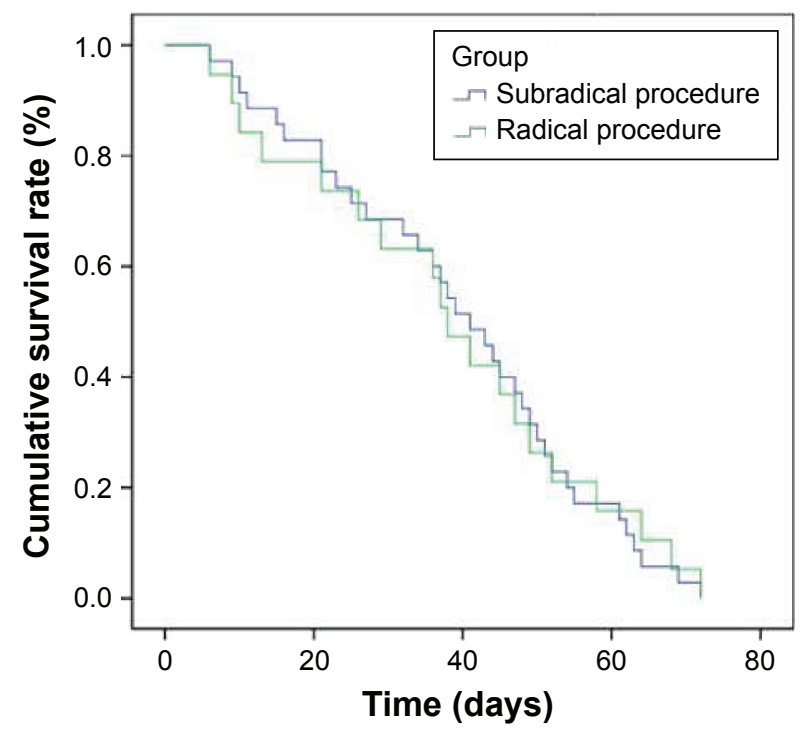

Figure I Clinical outcome of patients receiving different surgical procedures. Note: No significant differences were observed in overall survival between the two surgical groups. on patient's general condition, tumor invasion extent, and tumor malignancy. For stage II endometrial carcinomas, the extent of surgical dissection had not reached an agreement. There were two types of surgeries: subradical hysterectomy or radical hysterectomy, with simultaneous bilateral salpingooophorectomy and pelvic and paraaortic lymphadenectomy. So far, there has been no consensus on which procedure should be adopted.

In our study, we first compared the surgical outcomes according to the procedures and found no significant differences in operative time, estimated blood loss, and hospital stay between subradical and radical procedures.

Then we focused on the postoperative complications related to specific procedures. The early complications were defined as those occurring during the first postoperative 8 weeks. Most of the complications were found in the urinary system and the pelvic lymph system, and the fewer ones were observed in the lymphatic system, the nervous system, and the incision site, similar to two previous studies. ${ }^{10,11}$ The late complications referred to those occurring 8 weeks after surgical treatment, mainly being the extension or sequelae of the early complications, such as sustained pelvic lymphocyst, leg weakness due to injury of genitofemoral nerve, and vaginal dryness and coital bleeding due to removal of ovaries.

The rate of early postoperative complications in patients who underwent subradical surgical procedure was significantly lower than that in patients who underwent radical surgery, whereas no significant difference was observed

Table 4 Clinical outcome of patients after surgery

\begin{tabular}{lll}
\hline Variables & $\begin{array}{l}\text { Subradical } \\
\text { hysterectomy }(\mathbf{n}=\mathbf{3 5})\end{array}$ & $\begin{array}{l}\text { Radical } \\
\text { hysterectomy }(\mathbf{n}=\mathbf{1 9})\end{array}$ \\
\hline Overall survival rate & $100 \%(35 / 35)$ & $100 \%(19 / 19)$ \\
Relapse rate & $5.71 \%(2 / 35)$ & $5.26 \%(1 / 19)$ \\
\hline
\end{tabular}


in the rate of late postoperative complications. The results suggested that subradical hysterectomy produced less early postoperative complications compared with radical hysterectomy.

In our series, three patients presented with urinary retention and two patients with secondary urinary tract infection. Patients who underwent radical hysterectomy plus pelvic lymphadenectomy tend to have dysfunction of bladder detrusor to varying degrees after surgery due to injury of pelvic blood supply and autonomic nerves, which eventually led to postoperative urine retention and secondary urinary tract infection. ${ }^{12-14}$ Sparing nerves and reducing intraoperative hemorrhage ${ }^{15,16}$ and indwelling urinary catheter for 7-14 days and bladder training are very important in helping patients to restore the bladder voiding function. ${ }^{17}$

In our study, a total of nine patients $(16.7 \%)$ were found to have pelvic lymphocyst after surgery. Lymphocele, defined as a collection of lymphatic fluid in the retroperitoneal space, is a complication that occurs following lymphadenectomy due to gynecological or urological malignancy and is believed to result from operative disruption and inadequate ligation of lymphatic trunks. ${ }^{18}$ Lymphocyst was first reported in 1955 by Mori describing the occurrence of 68 lymphoceles following radical hysterectomy and lymphadenectomy for cervical cancer. ${ }^{19}$ The majority of lymphocysts are asymptomatic and are often an incidental finding during postoperative or routine follow-up without clinical impact, with a reported incidence ranging from $1 \%$ to $58 \%{ }^{20}$ A symptomatic lymphocyst is a rare event occurring in only $5.8 \%$ of patients, which may compress adjacent structures consequently causing pain, hydronephrosis, urinary urgency, or thrombosis. ${ }^{21}$ Secondary infection is the most serious complication, and studies have found that the infection incidence rate is $<1 \%$. ${ }^{22}$ In our series, one of the nine patients $(11.1 \%)$ had lymphocyst infection presenting with fever and abdominal pain and underwent puncture and drainage under the supervision of ultrasound for four times. Antibiotics are useful in the treatment of infected lymphocysts, particularly in cases where surgical drainage is insufficient because of cysts with septae or high viscosity fluid. ${ }^{23}$

Deep vein thrombosis (DVT) and subsequent pulmonary embolism represent potentially lethal perioperative complications associated with major pelvic or abdominal surgeries. ${ }^{24}$ DVT is known to occur in the postoperative period or during the course of postoperative therapy in $9.8 \%-57.1 \%$ of patients with endometrial cancer. ${ }^{25}$ In our study, one patient receiving radical hysterectomy complained of ache in the left leg 3 days after surgery and found DVT after ultrasound examination. This might be attributed to extended operation time, long time of venous flow retardation, injury of venous walls, and abnormal coagulation function. ${ }^{26}$ Fortunately, pulmonary embolism did not occur after therapeutic anticoagulation treatment. Starting activity after surgery as early as possible and prophylactic antithrombotic medication could effectively reduce the risk of DVT.

Neuropathy is not a rare postoperative complication after lymphadenectomy, and the most common injured nerve is the genitofemoral nerve. Patients often complain of numbness of lower limb after surgery. Usually, the numbness of lower limb would disappear after a period of recovery exercise. However, in our study, two patients with injury of obturator nerve or genitofemoral nerve presented with leg weakness after the early 8 weeks. They restored $\sim 80 \%$ of their original level of muscle strength.

For premenopausal patients, removal of bilateral ovaries had an effect on the secretion of sex hormones. Patients had menopausal symptoms, such as hot flash, night sweating, descent of sexuality, emotional fluctuation, etc. In our study, 13 patients did not yet experience menopause and two of them complained of vaginal dryness. However, hormone replacement therapy is not recommended since estradiol may increase the risk of relapse.

Furthermore, comparing the effects of surgical intervention on the prognosis of patients, we analyzed the overall survival as well as the relapse and death of patients who have received either subradical procedures or radical procedures. Our results showed that there were no significant differences in overall survival and relapse rate between the two surgical groups. Our data suggested that surgical dissection extent did not affect the clinical prognosis of patients with stage II endometrial carcinoma.

\section{Conclusion}

In conclusion, for stage II endometrial carcinoma, subradical hysterectomy presented with less early postoperative complications and similar survival duration and recurrence compared with radical hysterectomy and should be advocated in clinical treatment.

\section{Acknowledgment}

This study was supported by the National Natural Science Foundation of China (No 81402140).

\section{Disclosure}

The authors report no conflicts of interest in this work. 


\section{References}

1. Siegel R, Naishadham D, Jemal A. Cancer statistics, 2012. CA Cancer J Clin. 2012;62(1):10-29.

2. Creasman WT, Odicino F, Maisonneuve P, et al. Carcinoma of the corpus uteri. Int J Gynaecol Obstet. 2003;83(suppl 1):79-118.

3. Kueck AS, Gossner G, Burke WM, et al. Laparoscopic technology for the treatment of endometrial cancer. Int J Gynecol Obstet. 2006;93(2): $176-181$.

4. Eltabbakh GH, Mount SL. Laparoscopic surgery dose not increase the positive peritoneal cytology among women with endometrial carcinoma. Gynecol Oncol. 2006;100(2):361-364.

5. Galaal K, Bryant A, Fisher AD, et al. Laparoscopy versus laparotomy for the management of early stage endometrial cancer. Cochrane Database Syst Rev. 2012;9:CD006655.

6. Walker JL, Piedmonte MR, Spirtos NM, et al. Laparoscopy compared with laparotomy for comprehensive surgical staging of uterine cancer: Gynecologic Oncology Group Study LAP2. J Clin Oncol. 2009; 27(32):5331-5336.

7. Kroft J, Li Q, Saskin R, et al. Trends over time in the use of laparoscopic hysterectomy for the treatment of endometrial cancer. Gynecol Oncol. 2015;138(3):536-541.

8. Yim GW, Kim SW, Nam EJ, et al. Perioperative complications of robot-assisted laparoscopic surgery using three robotic arms at a single institution. Yonsei Med J. 2015;56(2):474-481.

9. Calle EE, Rodriguez C, Walker-Thurmond K, et al. Overweight, obesity, and mortality from cancer in a prospectively studied cohort of U.S. adults. $N$ Engl J Med. 2003;348(17):1625-1638.

10. Piovano E, Fuso L, Poma CB, et al. Complications after the treatment of endometrial cancer: a prospective study using the French-Italian glossary. Int J Gynecol Cancer. 2014;24(3):418-426.

11. Backes FJ, Rosen M, Liang M, et al. Robotic hysterectomy for endometrial cancer in obese patients with comorbidities: evaluating postoperative complications. Int J Gynecol Cancer. 2015;25(7):1271-1276.

12. Zhang P, Hu WL, Cheng B, et al. A systematic review and meta-analysis comparing immediate and delayed catheter removal following uncomplicated hysterectomy. Int Urogenecol. 2015;26(5):665-674.

13. Trautner BW, Hull RA, Darouiche RO. Prevention of catheter-associated urinary tract infection. Curr Opin Infect Dis. 2005;18(1):37-41.
14. Cardosi RJ, Cardosi RP, Grendys EC Jr, et al. Infectious urinary tract morbidity with prolonged catheterization after radical hysterectomy. Am J Obstet Gynecol. 2003;189(2):380-383.

15. Aoun F, van Velthoven R. Lower urinary tract dysfunction after nervesparing radical hysterectomy. Int Urogenecol J. 2015;26(7):947-957.

16. Manesch F. Urodynamic study of bladder function following nerve sparing radical hysterectomy. J Gynecol Oncol. 2014;25(3):159-161.

17. Fanfani F, Costantini B, Mascilini F, et al. Early postoperative bladder training in patients submitted to radical hysterectomy: is it still necessary? A randomized trial. Arch Gynecol Obstet. 2015;291(4):883-888.

18. Charoenkwan K, Kietpeerakool C. Retroperitoneal drainage versus no drainage after pelvic lymphadenectomy for the prevention of lymphocyst formation in patients with gynecological malignancies. Cochrane Database Syst Rev. 2014;6:CD007387.

19. Mori N. Clinical and experimental studies on the so-called lymphocyst which develops after radical hysterectomy in cancer of the uterine cervix. J Jpn Obstet Gynecol Soc. 1955;2(2):178-203.

20. Zikan M, Fischerova D, Pinkavova I, et al. A prospective study examining the incidence of asymptomatic and symptomatic lymphoceles following lymphadenectomy in patients with gynecological cancer. Gyencol Oncol. 2015;137(2):291-298.

21. Achouri A, Huchon C, Bats AS, et al. Complications of lymphadenectomy for gynecologic cancer. Eur J Surg Oncol. 2013;39(1):81-86.

22. Kavallaris A. Management of symptomatic pelvic lymphocyst after radical pelvic or pelvic and paraaortic lymphadenectomy for cervical and endometrial cancer. Gynecol Surg. 2009;6:345-349.

23. Kawamura I, Hirashima Y, Tsukahara M, et al. Microbiology of pelvic lymphocyst infection after lymphadenectomy for malignant gynecologic tumors. Surg Infect (Larchmt). 2015;16(3):244-246.

24. Turpie AG, Chin BS, Lip GY. Venous thromboembolism: pathophysiology, clinical features, and prevention. BMJ. 2002;325(7369): 887-890.

25. Satoh T, Matsumoto K, Uno K, et al. Silent venous thromboembolism before treatment in endometrial cancer and the risk factor. Br J Cancer. 2008;99(7):1034-1039.

26. Wang X, Fu S, Freedman RS, et al. Venous thromboembolism syndrome in gynecological cancer. Int $J$ Gynecol Cancer. 2006;16(suppl 1): $458-471$.
OncoTargets and Therapy

\section{Publish your work in this journal}

OncoTargets and Therapy is an international, peer-reviewed, open access journal focusing on the pathological basis of all cancers, potential targets for therapy and treatment protocols employed to improve the management of cancer patients. The journal also focuses on the impact of management programs and new therapeutic agents and protocols on

\section{Dovepress}

patient perspectives such as quality of life, adherence and satisfaction The manuscript management system is completely online and includes a very quick and fair peer-review system, which is all easy to use. Visit http://www.dovepress.com/testimonials.php to read real quotes from published authors. 